\title{
Atypical malignant late infective endocarditis of Melody valve
}

\author{
Mehul Patel, MD, ${ }^{\mathrm{a}}$ Laurence Iserin, MD, ${ }^{\mathrm{b}}$ Damien Bonnet, MD, PhD, ${ }^{\mathrm{a}, \mathrm{c}}$ and \\ Younes Boudjemline, MD, PhD, ${ }^{\mathrm{a}, \mathrm{b}, \mathrm{c}}$ Paris, France
}

The Melody transcatheter pulmonary valve (Medtronic, Inc, Minneapolis, Minn) is approved for interventional treatment of dysfunctional right ventricular outflow tract (RVOT) conduits as an alternative to surgical replacement. ${ }^{1}$ We report a series of 4 patients with infective endocarditis of a Melody valve. The data are derived from the REVALV trial, evaluating outcomes after Melody valve implantation in France.

From the Centre de Référence Malformations Cardiaques Congénitales Complexes-M3C, ${ }^{\text {a }}$ Necker Hospital for Sick Children, Assistance Publique des Hopitaux de Paris, Pediatric Cardiology, Paris, France; the Centre de Référence Malformations Cardiaques Congénitales Complexes-M3C, ${ }^{\mathrm{b}}$ George Pompidou European Hospital, Assistance Publique des Hopitaux de Paris, Unit for Adults with Congenital Heart Defects, Paris, France; and the Université Paris Descartes, ${ }^{\mathrm{c}}$ Sorbonne Paris Cité, Paris, France.

Funding provided by Ministry of Health (STIC, REVALV).

Disclosures: Authors have nothing to disclose with regard to commercial support.

Received for publication Nov 16, 2011; revisions received Dec 16, 2011; accepted for publication Jan 4, 2012; available ahead of print Jan 30, 2012

Address for reprints: Younes Boudjemline, MD, PhD, Hôpital Necker Enfants Malades, Cardiologie Pédiatrique, 149 rue de Sèvres, 75015 Paris Cedex, France (E-mail: younes.boudjemline@nck.aphp.fr).

J Thorac Cardiovasc Surg 2012;143:e32-5

$0022-5223 / \$ 36.00$

Copyright $\odot 2012$ by The American Association for Thoracic Surgery

doi:10.1016/j.jtcvs.2012.01.006

\section{CLINICAL SUMMARIES \\ Patient 1}

A 14-year-old boy with a Melody valve inserted for a hemodynamically dysfunctional Carpentier-Edwards conduit (Edwards Lifescience, Irvine, Calif) returned after 11.9 months with low-grade fever, positive blood cultures, and acute right heart failure. Echocardiogram showed severe pulmonary valvular obstruction with a right ventricular (RV) systolic pressure of $121 \mathrm{~mm} \mathrm{Hg}$ (Table 1). On an emergency basis, a bare metal stent was inserted with good hemodynamic results followed by surgical correction after 6 weeks (Table 2). Surgical examination showed patent but exposed stent struts with no fracture. The cusps were filled by large, fleshy, sessile, easily retrievable vegetations. Histologic examination showed voluminous fibrin strands with no signs of leaflet destruction (Figure 1). After prolonged postoperative antibiotics, the patient is now doing well with no recurrence at 3 months' follow-up.

\section{Patient 2}

An 18-year-old woman with surgically corrected tetralogy of Fallot using Hancock 16-mm conduit (Medtronic, Inc) underwent insertion of a Melody valve with good

TABLE 1. Baseline and infective endocarditis characteristics

\begin{tabular}{|c|c|c|c|c|}
\hline & Patient 1 & Patient 2 & Patient 3 & Patient 4 \\
\hline Age at presentation (y) & 14 & 18 & 26 & 11 \\
\hline Gender & Male & Female & Male & Male \\
\hline Underlying diagnosis & Aortic stenosis (Ross procedure) & TOF with PA & TOF with PA & $\begin{array}{l}\text { TOF with absent } \\
\text { pulmonary valve }\end{array}$ \\
\hline $\begin{array}{l}\text { Indication for PPVI, (RVOT/pulmonary } \\
\text { valve stenosis/insufficiency) }\end{array}$ & Mixed & Obstruction & Mixed & Obstruction \\
\hline RVOT characteristics and size & Carpentier-Edwards $20 \mathrm{~mm}$ & Hancock $16 \mathrm{~mm}$ & Hancock $18 \mathrm{~mm}$ & VenPro* $16 \mathrm{~mm}$ \\
\hline PPVI-IE time (mo) & 11.9 & 28.3 & 2.6 & 9.3 \\
\hline Prior IE & None & None & None & None \\
\hline Presentation & RVOT obstruction & $\begin{array}{l}\text { RVOT obstruction } \\
\quad+\text { RV failure }\end{array}$ & $\begin{array}{l}\text { RVOT obstruction } \\
\quad+\mathrm{RV} \text { failure }\end{array}$ & MAS \\
\hline Abrupt aspirin discontinuation & No & Yes & Yes & Yes \\
\hline Obvious source & None & None & None & None \\
\hline Involvement of other valves & Aortic valve & None & None & None \\
\hline $\begin{array}{l}\text { Location of vegetations ( } \mathrm{RVOT} \text { side/Pa } \\
\text { side) }\end{array}$ & Pa side & Pa side & Pa side & $\mathrm{Pa}$ side \\
\hline Destruction/abscess/fistulization & None & None & None & None \\
\hline Pulmonary valvular gradient & Severe & Severe & Severe & Moderate \\
\hline Microorganism from blood culture & Strep sanguis & Strep sanguis & Staph epidermidis & Strep mitis \\
\hline Embolic phenomenon & None & None & None & None \\
\hline
\end{tabular}

TOF, Tetralogy of Fallot; $P A$, pulmonary atresia; $P P V I$, percutaneous pulmonary valve insertion; $R V O T$, right ventricular outflow tract; $I E$, infective endocarditis; $M A S$, macrophage activation syndrome; $R V$, right ventricular; $P a$, pulmonary artery. *VenPro Corporation, Irvine, Calif. 
TABLE 2. Therapy and outcomes

\begin{tabular}{|c|c|c|c|c|}
\hline & Patient 1 & Patient 2 & Patient 3 & Patient 4 \\
\hline Response to antibiotics & Poor & None & None & Good \\
\hline Other therapies & Emergency BMS to relieve RVOT obstruction & - & - & - \\
\hline Surgery & $\begin{array}{l}\text { RVOT conduit replacement with } \\
\text { aortic valve replacement }\end{array}$ & Died immediately after surgery & Died before surgery & $\begin{array}{l}\text { RVOT conduit } \\
\text { replacement }\end{array}$ \\
\hline Outcome & Alive & $\begin{array}{l}\text { Died within } 24 \mathrm{~h} \text { of admission } \\
\text { in the operating room owing } \\
\text { to } \mathrm{RV} \text { failure/MOF }\end{array}$ & $\begin{array}{l}\text { Died with } 24 \mathrm{~h} \text { of admission } \\
\text { owing to } \mathrm{RV} \text { failure/VF }\end{array}$ & Alive \\
\hline
\end{tabular}

$B M S$, Bare metal stent; $R V O T$, right ventricular outflow tract; $R V$, right ventricle; $V F$, ventricular fibrillation; $M O F$, multiorgan failure.

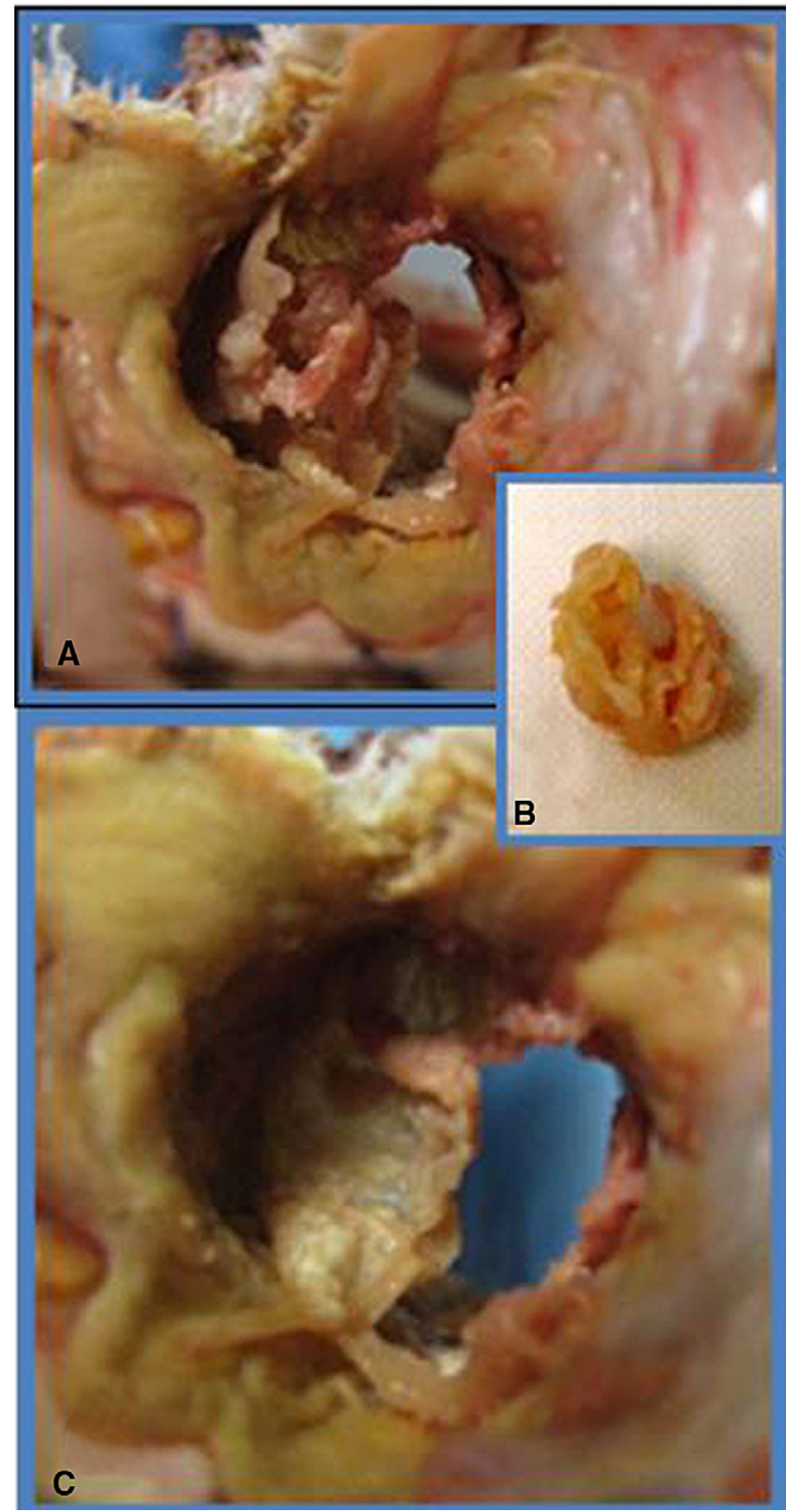

FIGURE 1. Ex vivo images of right ventricular outflow tract specimen of patient 1 . The bare metal stent has been retrieved and is not shown. A, The explanted Melody valve with very large vegetation completely filling the cusps. B, Image of the sessile vegetation. C, Appearance of the prosthetic valve leaflets after removal of the vegetation. immediate outcome. After 28.3 months, the patient had a 1-week history of low-grade fever, severe RV failure, and shock liver. The RV systolic pressure by Doppler was $141 \mathrm{~mm} \mathrm{Hg}$ and the RVOT gradient was $121 \mathrm{~mm} \mathrm{Hg}$. Emergency surgery was performed with extracorporeal membrane oxygenation support, but the patient died of multiorgan failure. Autopsy examination showed wellpreserved stent struts with large, loose, obstructive vegetations filled with fibrinous material.

\section{Patient 3}

A 26-year-old man was admitted 2.6 months after Melody valve insertion with a 10-day history of low-grade fever, light headedness, and acute right heart failure. An echocardiogram showed large, obstructive pulmonary valve vegetations with severe RV dysfunction. Surgery was planned the next day; however, the patient died on the night of presentation owing to relentless RV failure and ventricular fibrillation. Autopsy examination showed large, pink vegetations in 2 of 3 cusps and a well-preserved Melody valve stent frame (Figure 2). Histologic examination confirmed infective endocarditis with voluminous thrombus and microorganisms.

\section{Patient 4}

An 11-year-old boy with insertion of a Melody valve for severe RVOT obstruction was seen after 9.3 months with fever and signs and symptoms of macrophage activation syndrome. An echocardiogram showed moderate-sized vegetations on the pulmonary valve with isosystemic RV pressures and no RV failure. Surgical revision with a Contegra 22-mm conduit (Medtronic, Inc) was performed with rapid improvement and no recurrence after 8 years of follow-up. Histologic examination revealed signs of calcification in 1 leaflet, inflammation and extensive polymorphonuclear neutrophilic infiltration in another, and thrombus formation in the third (Figure 3).

\section{DISCUSSION}

Prosthetic valve endocarditis has been described to occur early or late after surgery. There are anecdotal reports of infective endocarditis of Melody valves. ${ }^{2,3}$ The late 


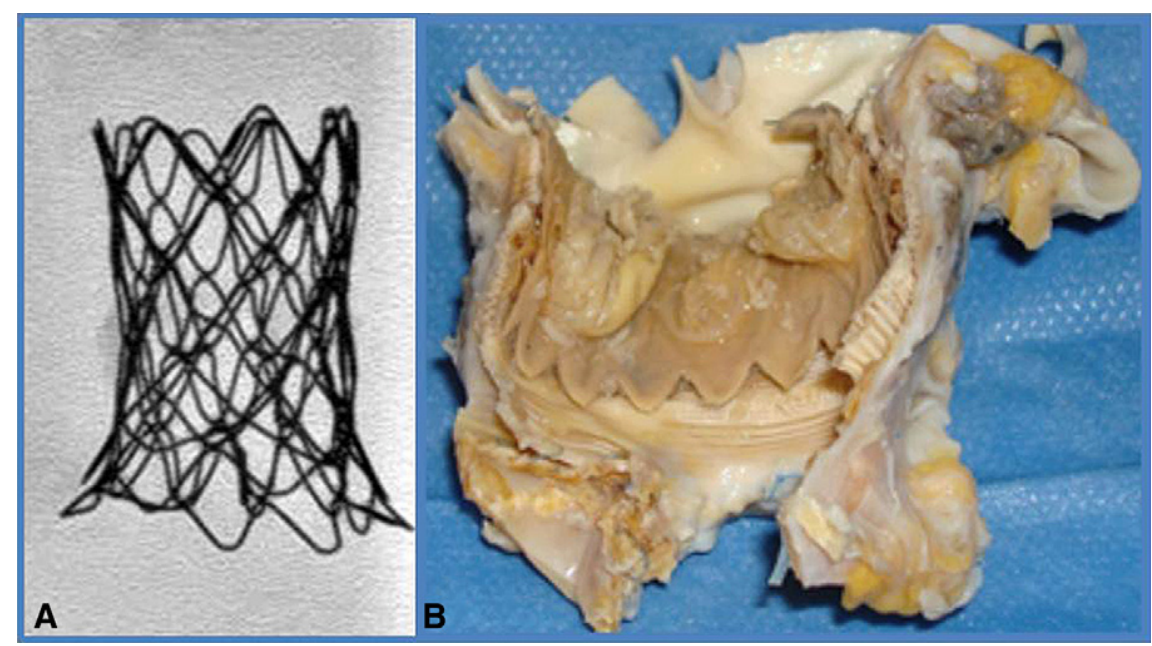

FIGURE 2. A, Ex vivo x-ray image of the conduit from patient 3 showing well-preserved frame of the bare metal stent struts with no fracture or collapse. B, Macroscopic examination of the slit open right ventricular outflow tract specimen showing the large vegetations filling up 2 of 3 cusps.

and near similar acute presentation, abrupt aspirin discontinuation, and similar pathologic finding highlight the possibility of a common pathophysiologic pathway. It is difficult to identify definitive predictors of Melody valve endocarditis from this case series; however, some notably common factors deserve special attention (Figure 4). Incomplete opening of the RVOT conduit during percutaneous pulmonary valve insertion with resultant residual gradient may be the most important inciting factor disrupting laminar flow causing eccentric turbulence and thrombogenesis. The voluminous thrombus seems to be the first step in the pathophysiology. Thrombus formation has been noted to occur with Contegra conduits used in various surgical situations. ${ }^{4}$ Recommendations have been made to continue aspirin after percutaneous pulmonary valve insertion for at least 6 months. Our policy is to continue aspirin lifelong in all patients; however, 3 of 4 patients with poor compliance abruptly discontinued aspirin. Further seeding by microorganisms with rapid progression is not well understood.
A prior report of infective endocarditis on bovine conduits may suggest possible tropism of microorganisms to the bovine material used for making the Melody valve. ${ }^{5}$ The inherent biological characteristics of the Contegra valve and/or its method of preparation may be contributing to the thrombogenicity and possible tropism for microorganisms.

\section{CONCLUSIONS}

Late infective endocarditis with the Melody valve raises some safety concerns, independent of the quality of device and the efficacy of the procedure. Additional input from multicentric, large registries may identify the true incidence and annual risk in such patients. Strict antibiotic prophylaxis with extensive preprocedure infectious disease evaluation must be done in all patients. Urgent intervention to relieve acute obstruction is likely to reduce mortality and should always be considered along with aggressive management of infective endocarditis.

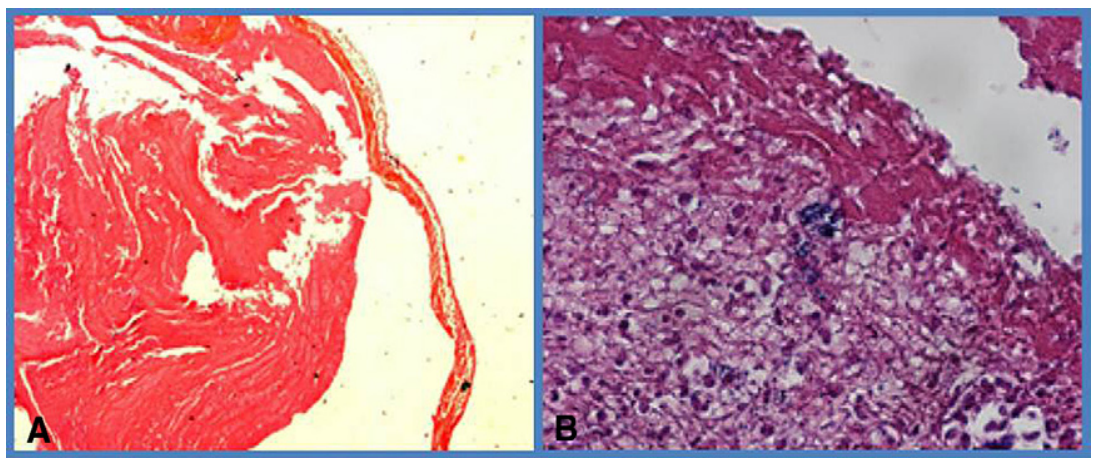

FIGURE 3. Histologic appearance of vegetation from patient 3; voluminous acellular thrombus (A) and extensive stuffing of polymorphonuclear neutrophils (B). 


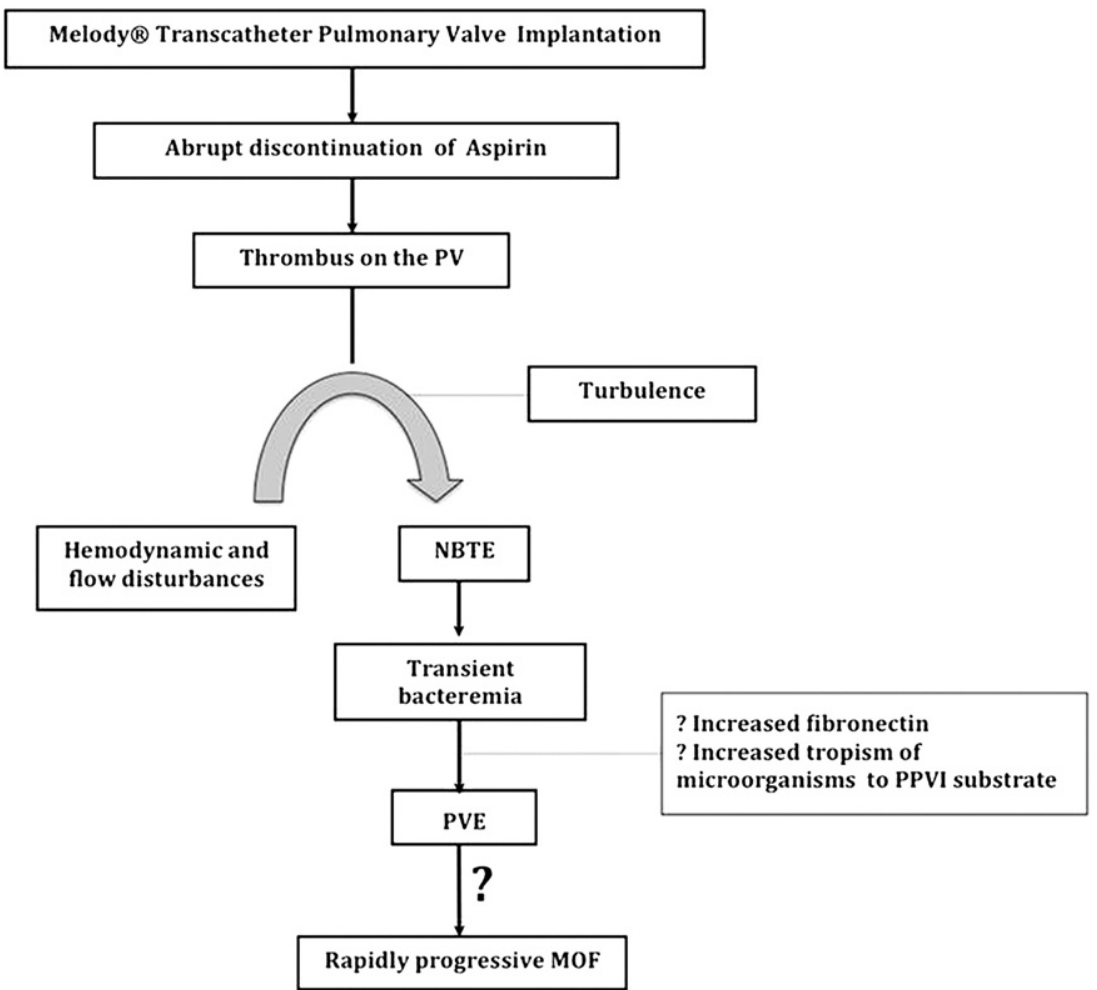

FIGURE 4. Proposed pathophysiologic mechanism for infective endocarditis of the Melody valve. $P V$, Pulmonary valve; $N B T E$, nonbacterial thrombotic endocarditis; $P P V I$, percutaneous pulmonary valve insertion; $P V E$, prosthetic valve endocarditis; $M O F$, multiorgan failure.

\section{References}

1. Lurz P, Coats L, Khambadkone S, Nordmeyer J, Boudjemline Y, Schievano S, et al. Percutaneous pulmonary valve implantation: impact of evolving technology and learning curve on clinical outcome. Circulation. 2008;117: 1964-72.

2. Lurz P, Bonhoeffer P, Taylor AM. Percutaneous pulmonary valve implantation: an update. Expert Rev Cardiovasc Ther. 2009;7:823-33.
3. Atamanyuk I, Raja SG, Kostolny M. Bartonella henselae endocarditis of percutaneously implanted pulmonary valve: a case report. J Heart Valve Dis. 2011;20:94-7.

4. Boudjemline Y, Bonnet D, Massih TA, Agnoletti G, Iserin F, Jaubert F, et al. Use of bovine jugular vein to reconstruct the right ventricular outflow tract: early results. J Thorac Cardiovasc Surg. 2003;126:490-7.

5. Bajraktari G, Olloni R, Daullxhiu I, Ademaj F, Vela Z, Pajaziti M. MRSA endocarditis of bovine Contegra valved conduit: a case report. Cases J. 2009;2:57.

\title{
Iatrogenic left internal thoracic artery to left subclavian vein fistula after excimer laser pacemaker lead extraction
}

\author{
Melissa M. Anastacio, MD, ${ }^{\mathrm{a}}$ Mario Castillo-Sang, MD, ${ }^{\mathrm{a}}$ Timothy W. Smith, MD, DPhil, ${ }^{\mathrm{b}}$ \\ Nael Saad, MD, ${ }^{\mathrm{c}}$ and Jennifer S. Lawton, MD, ${ }^{\mathrm{a}}$ St Louis, Mo
}

\footnotetext{
From the Division of Cardiothoracic Surgery, ${ }^{\mathrm{a}}$ Department of Surgery, the Division of Cardiovascular Medicine, ${ }^{\mathrm{b}}$ Department of Medicine, and the Mallinckrodt Institute of Radiology, ${ }^{\mathrm{c}}$ Washington University School of Medicine, St Louis, Mo.

Disclosures: Authors have nothing to disclose with regard to commercial support.

Received for publication Dec 15, 2011; accepted for publication Jan 4, 2012; available ahead of print Feb 3, 2011.

Address for reprints: Jennifer S. Lawton, MD, Washington University School of Medicine, 660 S Euclid Ave, Campus Box 8234, St Louis, MO 63110 (E-mail: lawtonj@wustl.edu).

J Thorac Cardiovasc Surg 2012;143:e35-7

$0022-5223 / \$ 36.00$

Copyright (c) 2012 by The American Association for Thoracic Surgery

doi:10.1016/j.jtcvs.2012.01.014
}

Percutaneous methods of pacemaker and defibrillator lead extraction have decreased the morbidity associated with pacemaker management through traditional open chest surgery but carry their own serious complications, including bleeding, cardiovascular injury, hemothorax or pneumothorax, and even death. We report here a rare case of iatrogenic arteriovenous fistula formation after percutaneous pacemaker lead extraction with an excimer laser. 